www.ocula.it • ISSN 1724-7810 • Vol 21, No 24 (October 2020) • DOI: 10.12977/ocula2020-44

Quando è design When is Design Quand c'est du design

a cura di Michela Deni e Dario Mangano

\title{
È design della comunicazione
}

Valeria Bucchetti

Dipartimento di Design, Politecnico di Milano, Milano, IT

valeria.bucchetti@polimi.it

\begin{abstract}
The contribution intends to limit the field of reflection to Communication Design. We therefore propose to reformulate the question: "When is Communication Design"? When can we talk about Communication Design and what are the conditions and processes that allow us to support it?

To take care of them means to refocus the different levels in which the field is articulated: from that of the communicative artefacts, in their prosthetic dimension, to that of the systems that organize them, starting from directorial logic, coming to trace implicitly the profile of the communication designer.
\end{abstract}

\section{Keywords}

Communication Design; Artefacts; Systems; Design Culture.

\section{Contents}

1. Premesse all'interrogativo

2. Le ragioni delle parole

3. Quando è cultura del progetto

Bibliografia 
Vol 21, No 24 (October 2020) • DOI: 10.12977/ocula2020-44

Valeria Bucchetti | È design della comunicazione

\section{Premesse all'interrogativo}

L'interrogativo "Quando è design" sembra far perdere i confini. A questa domanda provo quindi a rispondere circoscrivendo innanzitutto il mio contributo al design della comunicazione.

Come è noto, quantomeno in Italia, si vede associare alla parola design, usata senza alcuna specificazione, la nozione di industrial design, così come De Fusco (2008) argomenta in premessa al suo testo Parodie del design.

Se si vuole parlare di design secondo una prospettiva disciplinare, e non dell'accezione che la conversazione quotidiana ha attribuito al termine, distorcendone il significato unicamente verso una dimensione estetizzante, il design ha sempre la necessità di essere connotato attraverso un'etichetta attributiva, attraverso un termine di specificazione a partire dal quale lo si declina e che ne determina una precisa definizione di campo (De Fusco 1985, 2008; Trabucco 2015).

Il Design della comunicazione, in questo senso, afferma la propria esistenza, il proprio ambito di azione, ma anche il proprio specifico disciplinare, all'interno del più vasto campo del Design che rimanda, invece, a svariati contenuti possibili a cura della progettualità.

E fondamentale precisare come alcune trasformazioni avvenute negli ultimi decenni, anche sul piano istituzionale - attraverso la costituzione di corsi di laurea -, abbiano reso esplicita l'articolazione del campo in ambiti disciplinari autonomi, seppure tra loro in dialogo costante. Quando parliamo di design degli interni, design della moda, design del prodotto, design della comunicazione facciamo riferimento ad aree del progetto con un proprio baricentro specifico, ciascuna delle quali vede coinvolti nuclei distinti di saperi disciplinari. E questo vale per i saperi storico-critici, tra loro differenti, dalla storia del prodotto industriale per design del prodotto, alla storia della grafica per design della comunicazione; così come per le aree a carattere tecnologico, sino alle conoscenze che coinvolgono le tecniche di produzione. Allo stesso modo, all'interno di ciascun ambito disciplinare vengono sviluppati sistemi artefattuali caratterizzati e caratterizzanti, che nascono da un processo progettuale radicato in quella precisa cultura del progetto. Una cultura del progetto improntata sulla dimensione autoriflessiva, propria delle discipline del design, ovvero sulla capacità della disciplina di riflettere su se stessa attraverso l'agire progettuale; capacità che non può, pertanto, prescindere dalle specificità dei saperi disciplinari che ne stanno alla base.

Fatta questa precisazione vorrei quindi provare a interrogarmi, e a declinare la domanda quando è design, proprio su una di queste aree, mettendo al centro il design della comunicazione, chiedendomi ‘quando’ è design della comunicazione. Quando il design della comunicazione si dà.

Per tracciare delle risposte ritengo indispensabile ripartire da alcune definizioni che consentono di perimetrare ciò di cui parliamo. Giovanni Lussu (2010), nella definizione elaborata per l'Enciclopedia Treccani, osserva come l'espressione design della comunicazione sia: 
Vol 21, No 24 (October 2020) • DOI: 10.12977/ocula2020-44

Valeria Bucchetti | È design della comunicazione

inscindibile dall'esistenza di una specifica categoria di operatori che in tale ambito si riconoscono e in quanto tali vengono riconosciuti dall'ambiente in cui sono attivi: l'associazione professionale europea di gran lunga più rappresentativa, la Chartered society of designers.

E sottolinea ciò che si intende per design della comunicazione:

nella terminologia italiana e negli assetti didattici e istituzionali, per design della comunicazione s'intende oggi (rispetto al termine più generico grafica, il cui significato tende ormai più verso l'ambito espressivo) la progettazione di artefatti comunicativi, in particolare di tipo visivo, svolta da operatori specializzati in presenza di precisi vincoli produttivi e con obiettivi più nettamente tesi agli aspetti di tipo funzionale, legati alla risoluzione di specifici problemi posti da determinate committenze, pubbliche o private.

Una definizione quindi molto chiara che traccia con una certa nettezza il campo, poiché delinea le condizioni per poterne parlare, alla quale possiamo affiancare un ulteriore tassello parafrasando ciò che negli anni Sessanta Tomás Maldonado sosteneva al congresso ICSID (Venezia, 1961) e che viene riportato nel suo testo Disegno industriale: un riesame. Possiamo asserire che progettare il formato comunicativo significa «coordinare, integrare e articolare tutti quei fattori che, in un modo o nell'altro, partecipano al processo costitutivo della forma del prodotto [dell'artefatto comunicativo]. E precisamente si allude tanto ai fattori relativi all'uso, fruizione e consumo individuale o sociale del prodotto (fattori funzionali, simbolici o culturali) quanto a quelli relativi alla sua produzione (fattori tecnico-economici, tecnico-costruttivi, tecnico-sistemici, tecnico-produttivi e tecnico-distributivi)» (Maldonado 1976: 9-10).

\section{Le ragioni delle parole}

Sebbene questo primo perimetro possa aiutarci a comprendere la questione, vorrei richiamare il fatto che l'espressione design della comunicazione si è affermata solamente negli ultimi decenni e che rappresenta la risultante di un processo evolutivo di grande importanza per l'intera area, il cui esito ha trovato in questa locuzione l'espressione della propria identità disciplinare.

Si tratta di una locuzione che sottende infatti molteplici nozioni e richiamarle ci aiuta a chiarirne la natura.

In primis vi è la nozione di grafica, col suo «richiamarsi al laboratorio pre e proto-storico della nascita della scrittura, del tracciare e dello scrivere» (Anceschi 1991: 56; vedi anche: Anceschi 1981; D’Ambrosio, Grimaldi, Lenza 1985; De Fusco 1985; Margolin 2002); vi è la nozione di Graphic design che, come sottolinea Anceschi, potrebbe essere la definizione dell'attività sistemica a partire da Gutenberg, da Manuzio, da Griffo; non ultima, la nozione di comunicazioni visive che non designa tanto una professionalità quanto un 
Vol 21, No 24 (October 2020) • DOI: 10.12977/ocula2020-44

Valeria Bucchetti | È design della comunicazione

campo sterminato della produzione e della fruizione di oggetti, di programmi, di beni e servizi della comunicazione, dell'emissione e della ricezione di messaggi, comprendendo inoltre tutte le strategie e tutte le tecniche, tutti gli artifici e tutti i semilavorati che concorrono alla loro realizzazione. (ibidem)

A queste nozioni va aggiunto poi

il termine di conio anglosassone Visual design (che potremmo tradurre progettazione visiva, e che quindi chiaramente tende a collocare il fenomeno nel quadro del raggruppamento delle discipline progettuali accanto al disegno industriale, all'architettura ecc.). (Anceschi 1988: 11)

La nozione di visual design (vedi Vitta 1999) risultava peraltro consolidata e assolutamente chiara nella sua portata già nel 1954 quando Nino Di Salvatore fondò a Novara la prima scuola di Design in Italia (la Scuola Politecnica di Design) - e poi a Milano nel 1970 -, quando attraverso di essa designava uno dei corsi biennali. Il corso di Visual design e il corso di Industrial Design articolavano infatti l'offerta didattica della Scuola proponendo due programmi e due piani di studio distinti, sebbene entrambi riferiti al "design" e declinati in base alle caratteristiche di ciascun ambito.

A esse si è aggiunta poi anche la nozione di sistemi grafici, che fu la denominazione della disciplina insegnata al Dams di Bologna negli anni Ottanta, intesa

come la teorizzazione dell'insieme dei metodi e dei sistemi della progettazione di comunicazioni visive e, contemporaneamente, come la disciplina della progettazione di sistemi più o meno complessi di comunicazione visiva (cioè sistemi di segnaletica, di notazione, di raffigurazione ecc.). (ibidem)

Accezione questa che, come lo stesso Anceschi ricorda in Monogrammi e figure (1988: 12) «ingloba quella originaria e ristretta di Graphic Systems, cioè lo studio della progettazione e dell'uso di sistemi integrati e altamente tecnologizzati di composizione, impaginazione e stampa».

Si tratta certamente di un quadro composito, all'interno del quale l'individuazione di campo della grafica non è un dato assoluto; un quadro costituito da una molteplicità di approcci e da numerose definizioni che vedono instabile la definizione stessa di grafica. Instabilità che sembra essere connaturata con l'oggetto di ricerca, con l'ambito di attività, con le concrete mutazioni di un fare che ne ha marcato il profilo anche nei decenni successivi.

Anceschi affermava:

Se tentiamo cioè di delineare un profilo preliminare e sommario dell'oggetto di indagine è difficile, anche se ci si sforzi di procedere schematicamente, è anzi estremamente difficile sfuggire al meno definitorio e più aperto dei procedimenti di definizione e cioè l'elencazione. (Anceschi 1988: 11-12) 
Vol 21, No 24 (October 2020) • DOI: 10.12977/ocula2020-44

Valeria Bucchetti | È design della comunicazione

Il "profilo" dell'ambito disciplinare del design della comunicazione racchiude cioè un vasto elenco non solo di tipologie artefattuali proprie di questa specifica area del design, ma anche una vasta area nella quale si svolgono:

una serie di azioni, di procedimenti e di operazioni di cui fanno parte ad esempio quelle del rappresentare e del presentare, del trasmettere e del tradurre, dell'esporre, del descrivere, decodificare e del simboleggiare.

E ciò si realizza col tramite di tecniche e tecnologie svariate, servendosi di sistemi e di metodi, ma anche di accorgimenti e di trucchi, e avviene con l'aiuto di strumenti e di attrezzi produttivi, nonché di macchinari fabbricativi ecc. Tutte queste azioni e operazioni confluiscono poi e si coagulano in un oggetto, o meglio, in una serie di oggetti materiali, destinati in prima istanza semplicemente a stimolare gli organi visivi di uno o molti destinatari, e più in generale diretti ad essere da essi per qualche motivo usati o più genericamente fruiti. (ibidem)

È tra la fine degli anni Ottanta e l'inizio degli anni Novanta che, dall'intersezione di questi termini, nasce e si afferma la nozione di Design della comunicazione. Ossia quando

il sistema della comunicazione e dell'informazione dispone di «una presenza generalizzata, di una diffusione capillare, di un assetto poderoso». Quando cioè è «l'industria della comunicazione e dell'informazione a porsi come traente nello scenario contemporaneo». (vedi: Carta del progetto grafico)

In questo momento di trasformazione fu determinante la Carta del Progetto grafico, la sua stesura e la sua divulgazione. Un documento la cui redazione venne affidata a una commissione di estensori formata da Giovanni Anceschi, Giovanni Baule, Gianfranco Torri, a sua volta espressa da un comitato formato, oltre che dai tre citati, anche da Gelsomino D'Ambrosio, Pino Grimaldi, Giancarlo Iliprandi, Giovanni Lussu, Alberto Marangoni, costituitosi in occasione della preassemblea nazionale AIAP ad Aosta il 24 giugno 1989.

Si tratta di un testo che per la sua contemporaneità ci aiuta ancora oggi a tracciare delle risposte in merito alla questione complessiva che ci stiamo ponendo. Innanzitutto la Carta riportava come sottotitolo "Tesi per un dibattito sul progetto della comunicazione", quindi letteralmente sul design della comunicazione. Il sottotitolo, inoltre, attraverso il termine tesi sottolineava e rendeva immediata la formula aperta con la quale la Carta intendeva caratterizzarsi. Potremmo dire, un modo per interrogarsi su "quando fosse design della comunicazione" e per aprire una discussione interna all'area estendendo gli esiti in un dibattito pubblico dichiarato da uno sviluppo a tesi.

Nel preambolo, il testo della Carta inquadrava il nuovo campo della grafica. Ciò fu allora fatto con un taglio pragmatico, giocato sull'evidenza imposta dal nuovo paesaggio comunicativo. Un primo argomento - in quel momento storico - è la constatazione del sistema della comunicazione come elemento chiave della contemporaneità e, a trent'anni di distanza, non possiamo che ri- 
Vol 21, No 24 (October 2020) • DOI: 10.12977/ocula2020-44

Valeria Bucchetti | È design della comunicazione

conoscere la centralità e l'attualità di questo passaggio che ha visto nel tempo un profondo rafforzamento del ruolo della comunicazione progettata.

Ma, come si evince da uno dei primi passaggi testuali, anche la presa d'atto in termini critici della conseguente invasività dei fenomeni comunicativi e l'indicazione della funzione e della responsabilità che la progettualità deve assumere.

Come ricorda Baule, in un saggio pubblicato sul numero della rivista AIS Design nel 2015 dedicato a "I designer e la scrittura nel Novecento", «è il primo pronunciamento che anticipa i passaggi che fanno della Carta un atto di design critico».

La presenza trasversale della grafica individua una dimensione estesa capace di trainare una pratica professionale diffusa: si tratta di un'estensione quantitativa e qualitativa, per supporti diversi e diversi campi applicativi che supera le forme tradizionali della grafica gutenberghiana. (Baule 2015: 9)

E se la Carta qui interviene come primo tracciamento di una mappatura dei campi applicativi della grafica, attraverso una sua rilettura attuale ci dà anche altri elementi per rispondere alla domanda "quando è design della comunicazione".

La Carta infatti affermava, sebbene lo facesse impiegando ancora il termine grafica, al quale noi oggi possiamo sostituire design della comunicazione, quanto la grafica fosse ormai una presenza trasversale. Il punto 1. della Carta dichiara che è là dove la cultura si fa editoria, è là dove i sistemi di trasporto si stanno informatizzando, interviene nell'assetto multimediale della politica. $\grave{E}$ presente non solo nella divulgazione ma anche nella modellazione della scienza, è in azione là dove il prodotto industriale interagisce con l'utilizzazione, $\grave{e}$ nella grande distribuzione dove il consumatore incontra la merce, è nello sport, nell'immagine delle grandi manifestazioni come nella loro diffusione massmediali ecc.

La Carta si pone dunque l'obiettivo di delineare un inquadramento di un'area della progettazione che intendeva riconoscersi come autonoma, rilanciando un ruolo e una funzione vissuti con ritardo nello stesso ambito della cultura del progetto. Allo stesso tempo essa

si conferma come un atto fondativo aperto, un ponte tra una radicata tradizione e le aspettative della rivoluzione digitale; marca una soglia storica e allarga i confini di campo culturale e di competenza disciplinare, puntando al riconoscimento di una figura professionale di riferimento. (op. cit: 14)

Getta le basi di quello che è andato poi configurandosi come l'attuale design della comunicazione.

In modo particolare fissa, sul piano dei saperi, della pratica e delle applicazioni progettuali un'accezione più estesa di progetto grafico, in grado di aprirsi ai sistemi multimediali, al design delle interfacce, alle forme di transmedialità. 
Vol 21, No 24 (October 2020) • DOI: 10.12977/ocula2020-44

Valeria Bucchetti | È design della comunicazione

La risultante della Carta sembra allora essere soprattutto una sorta di dichiarazione in filigrana di un profilo di designer che estende il proprio ambito di intervento ben oltre il sistema degli artefatti della grafica tradizionale e inizia ad attrezzarsi per la fase alta della rivoluzione digitale; rimarca un profilo della professione del progetto strettamente legato a una cultura e a un'identità disciplinare, consapevole di un ruolo di responsabilità all'interno del nuovo paesaggio comunicativo.

Apre un processo che, al di là di un dibattito strutturato, si attiverà successivamente nelle pratiche di programma come l'attuazione del primo sistema di formazione e di ricerca universitaria a rete nazionale dedicata al design della comunicazione. (ibidem)

È al Politecnico di Milano che nasce nel 1993 il primo corso di laurea in Disegno Industriale e, successivamente (nel 2003), in Design della comunicazione.

Ed è un anno dopo la pubblicazione della Carta che nell'assemblea nazionale straordinaria, l'Aiap (associazione nata nel 1945 come tecnici e Artisti Pubblicitari), associazione dei grafici, intendendo favorire e diffondere la valorizzazione e lo sviluppo della professione e della cultura del progetto grafico, adotta un nuovo statuto che, tra gli altri cambiamenti, influì anche sulla denominazione: la sigla AIAP fu mantenuta e venne aggiornata la sua specifica che fu trasformata in Associazione italiana Design della comunicazione visiva.

Oggi, nell'era di Wikipedia (cons. 27 luglio 2019), ricercando la voce "design della comunicazione" leggiamo:

Il design della comunicazione (in inglese communication design) è la branca del design che va a coprire tutti quegli ambiti in cui l'ideazione dei contenuti discende in buona parte dalla loro comunicabilità visuale.

\section{Quando è cultura del progetto}

Queste trasformazioni di cui ho dato conto, e i loro esiti, ci portano ad affermare che il Design della comunicazione si dà quando genera artefatti, si dà attraverso i formati della comunicazione, attraverso il sistema artefattuale nel suo complesso, là dove assume in forma implicita o esplicita una strategia comunicativa.

Ma dobbiamo anche sottolineare come la relazione tra artefatti comunicativi e design della comunicazione non sia una relazione biunivoca. La "produzione" di artefatti comunicativi, e più precisamente di protesi comunicative - secondo la prospettiva di Anceschi - non necessariamente implica "l'appartenenza" al Design della comunicazione. Possiamo trovarci di fronte a un artefatto comunicativo, ma non essere di fronte alla risultante di un'attività esercitata nel campo del design. Possiamo infatti affermare che il design delle comunicazione si dà attraverso gli artefatti, i formati della comunicazione, il sistema artefattuale... a patto che si operi all'interno della cultura del progetto e della sua dimensione sistemica: punto di intersezione di conoscenze storico-critiche, semiotiche, metodologiche, tecnico-produttive.

Un distinguo che si fa ancora più marcato nella contemporaneità in relazione all'espansione complessiva della produzione di azioni comunicative e di 
Vol 21, No 24 (October 2020) • DOI: 10.12977/ocula2020-44

Valeria Bucchetti | È design della comunicazione

artefatti che le sostanziano, alla disponibilità di strumenti diffusi che consentono a ciascun individuo di produrne, liberamente e indipendentemente da conoscenze disciplinari.

Ma soprattutto, proprio in relazione alle trasformazioni riferite alle tecnologie e agli apparati, come esplicita con grande chiarezza Giovanni Lussu sempre nel suo saggio, pubblicato per argomentare la nozione di design della comunicazione -, vediamo come:

Insieme alla parallela trasformazione degli assetti sociali e produttivi (con la progressiva scomparsa della "bottega" artigianale, ormai inadeguata alle esigenze del mercato), la tecnologia elettronica sta modificando profondamente il ruolo sociale del designer della comunicazione. È in atto, infatti, una sostanziale ridefinizione delle mansioni associate, perché gran parte di quelle tradizionali, com'è giusto che sia, vengono sempre più svolte da operatori di vario tipo che posseggono spesso solo una conoscenza di base nella gestione di un computer. Sono ormai disponibili software di facile acquisizione che assicurano uno standard qualitativo medio per la realizzazione di artefatti di uso corrente: pieghevoli promozionali, cataloghi commerciali, bollettini aziendali, siti web, prodotti di piccola editoria e così via vengono ormai prodotti da personale non specializzato.

[...] Il computer non fa che moltiplicare questa tendenza, non solo del tutto legittima ma senz'altro auspicabile: è evidente che, nel caso ne abbia la possibilità e gli strumenti, chi ha un'esigenza di comunicazione può ormai risolverla in modo appropriato ed economico. [...] Per quel che riguarda la realizzazione di artefatti comunicativi, il computer consente di gestire in proprio, fino all'utilizzazione di una stampante da ufficio, processi che in precedenza erano delegati, a pagamento, alla competenza di personale tecnico specializzato che ora rischia di apparire parassitario. (Lussu 2010)

Quella trattata da Lussu rappresenta una delle questioni "calde" che ricorrono al centro di riflessioni e dibattiti nel campo del progetto. Ricordo l'intervento di Beppe Chia, socio Aiap professionista quando, in una sua relazione presso l'università la Sapienza di Roma nel 2008 - Guardare, vedere, progettare: educare -, sottolineava l'urgenza di una riflessione sulla figura del progettista (del designer della comunicazione) in connessione a questi aspetti di semplificazione e standardizzazione dei dispositivi produttivi destinati a utenti generici, non "addetti ai lavori", ritenendo che molte delle funzioni e competenze del progettista fossero destinate a essere assunte direttamente dai software.

Ma se da un lato nella nostra civiltà i prodotti artefattuali della comunicazione sono divenuti così inconsapevolmente praticati con scopi differenti - basti pensare a quanto è avvenuto per esempio con la fotografia che, come osserva Zoja (2018: 11), «è di tutti sia nel senso che ognuno può vederne infinite, sia perché, prima con la diffusione degli apparecchi facili ed economici, poi con gli smartphone, scattarle è diventata un'abitudine quotidiana e universale»-, dall'altro, il compito del designer della comunicazione si pone su un piano differente, sia per ciò che riguarda gli obiettivi e i compiti progettuali, sia per ciò che ha a che fare con la responsabilità. E, anche in questo caso, 
Vol 21, No 24 (October 2020) • DOI: 10.12977/ocula2020-44

Valeria Bucchetti | È design della comunicazione

alcune risposte possiamo rintracciarle nella Carta del Progetto Grafico, in cui erano già contenute, e più precisamente al punto 6 .

Nei confronti dell'inquinamento prodotto da una comunicatività pletorica e da una complementare indifferenza per la cultura dell'immagine (risultato di una forma dell'industrializzazione dei processi comunicativi, dove l'industria massmediale e informazionale, prigioniera della ideologia dell'orientamento al mercato, produce vulcanicamente informazione), noi sottolineiamo le nuove responsabilità del progettista grafico [del designer della comunicazione]. Difendiamo il progetto della qualità nel campo della comunicazione visiva. Rivendichiamo nostre le responsabilità nei confronti dell'utenza. Competenza questa che è peraltro ciò che ci viene richiesta dalla committenza più avanzata. Noi dichiariamo pertanto il punto di vista dell'utenza fondamento costante del nostro operare.

Poniamo, inoltre, il massimo dell'attenzione, oltre che al risultato finale della comunicazione, anche a una presenza dei momenti strutturali e organizzativi della macchina della comunicazione... (Carta del Progetto Grafico 1989)

È interessante richiamare, in connessione con quanto appena affermato, ciò che veniva sostenuto da Di Salvatore nella documentazione prodotta per introdurre la propria proposta formativa nel campo del design.

Di Salvatore tra la fine degli anni Settanta e l'inizio degli anni Ottanta scriveva:

Il compito fondamentale del designer è quello di "umanizzare" il rapporto uomo-tecnica, proiettandolo e risolvendolo nel contesto culturale e sociale. L'ambito nel quale opera è costituito dalla tecnica (ideazione, necessità, mercato), dall'ergonomia (rapporto tra l'uomo e l'oggetto o la macchina; fattori della percezione per la comunicazione visiva), dalla società (sociologia, etica), dalla cultura (l'ambiente, la civiltà) e dalla proiezione nel futuro del design. È evidente che il primo requisito dei designer è l'alta coscienza professionale. (Di Salvatore 1981)

Ossia il design della comunicazione deve essere considerato, così come teorizzato da Victor Papanek (1983), uno strumento di trasformazione che deve accogliere punti di vista sociali ed etici, non necessariamente per le aree tematiche in cui si cala, ma attraverso la consapevolezza che il designer deve possedere in merito alle conseguenze sul piano trasformativo che il proprio agito implica.

Possiamo pertanto affermare che è design della comunicazione:

- dove vi è consapevolezza del processo e del suo governo, quindi quando si agisce con la consapevolezza di un metodo calato nella cultura progettuale;

- quando si è di fronte una scala elevata di complessità della comunicazione;

- laddove è implicata la nozione di sistema: da singoli artefatti a sistema;

- quando attraverso la progettazione parametrica si progettano i "comportamenti” e le configurazioni grafiche dei contenuti della comunicazione;

- quando l'azione progettuale è tesa a definire processi generativi (come nel caso delle identità generative) e i loro princìpi. 
Vol 21, No 24 (October 2020) • DOI: 10.12977/ocula2020-44

Valeria Bucchetti | È design della comunicazione

Così come la Carta recitava:

Consideriamo tra i nostri compiti principali quello di agire dentro ai sistemi che producono standard (dal design di caratteri ai progetti di simbologie segnaletiche, dai programmi di immagine coordinata alle strategie di comunicazione, dai software grafici a tutte quelle elaborazioni che serviranno in seconda istanza per produrre risultati finali). (Carta del Progetto Grafico 1989)

Oggi diciamo ancor più che è design della comunicazione all'interno di un campo in cui trova corpo la fusione fra diversi linguaggi, la graduale dissoluzione di codici comunicativi monolitici, in cui la cross-disciplinarietà tra saperi e culture, la convergenza tra produttori e consumatori, la confluenza di supporti e tecnologie in nuove piattaforme ibride, sono forme e dimensioni consolidate; ossia dove la competenza progettuale propria del designer della comunicazione è necessaria nella sua forma più elevata per affrontare e razionalizzare la dimensione emergente della trasformazione dei media e dei loro contenuti.

Quindi nulla a che vedere con quella "spinta corporativa di autoperpetuazione" che stava alla base delle critiche mosse da Giovanni Lussu quando accusava il settore di difesa corporativa, bensì la consapevolezza che la condizione per la crescita della cultura disciplinare pone al centro il governo dei processi, le pratiche traduttive, il ruolo registico del designer della comunicazione in relazione ai sistemi artefattuali che rendono attuabili le azioni comunicative.

\section{Bibliografia}

Anceschi, Giovanni

1981 Monogrammi e figure, Firenze, La casa Usher (seconda edizione riveduta e ampliata 1988).

Anceschi, Giovanni

1991 "Grafica, visual design, comunicazioni visive", in Castelnuovo E. (ed.). Storia del disegno industriale. 1919-199o, il dominio del design, Milano, Electa.

Baule, Giovanni

2015 La Carta del Progetto grafico venticinque anni dopo. Una rilettura, "AIS/ Design. Storia e ricerche", 6, 2015.

D’Ambrosio, Gelsomino; Grimaldi, Pino; Lenza, Cettina

1985 Sulla definizione di Grafica, "Grafica", 1, 1985, pp. 7-14.

De Fusco, Renato

1985 Storia del design, Bari, Laterza.

2008 Parodie del design, Torino, Allemandi.

Di Salvarore, Nino

1981 Scuola Politecnica di Design (catalogo della scuola). 
Vol 21, No 24 (October 2020) • DOI: 10.12977/ocula2020-44

Valeria Bucchetti | È design della comunicazione

Lussu, Giovanni

2010 "Design della comunicazione", Enciclopedia Treccani, <http://www.treccani. it/enciclopedia/design-della-comunicazione_\%28XXI-Secolo\%29/>.

Maldonado, Tomás

1976 Disegno industriale: un riesame, Milano, Feltrinelli.

Margolin, Victor

2002 The Politics of the Artificial: Essays on Design and Design Studies, Chicago, The University of Chicago Press.

Papanek, Victor

1983 Design for Human Scale, New York, Van Nostrand Reinhold.

Trabucco, Francesco

2015 Design, Torino, Bollati Boringhieri.

Vitta, Maurizio

1999 Il sistema delle immagini, Napoli, Liguori.

Zoja, Luigi

2018 Vedere il vero e il falso, Torino, Einaudi.

Carta del progetto grafico

1989 Pubblicata su LineaGrafica, 1 (gennaio), 1990.

Valeria Bucchetti è Professore ordinario di Disegno industriale presso il Dipartimento di Design (Politecnico di Milano); docente di Design della comunicazione nel Corso di laurea in Design della comunicazione, del quale è coordinatrice, e di Design della comunicazione e Culture di genere nei corsi di laurea Magistrale della Scuola del Design. Visual designer, laureata in DAMS (Università degli Studi di Bologna), dottore di ricerca in Disegno industriale. È stata socia AIAP (Associazione Italiana design della comunicazione visiva) dal 1989 al 2017; è stata membro dell'osservatorio dell'ADI Design Index; è membro del Collegio di Dottorato in Design e del comitato di redazione della collana di Design della comunicazione (FrancoAngeli). La sua attività di ricerca è orientata in particolare negli ambiti dell'identità visiva e dell'identità di prodotto, dell'identità di genere e degli stereotipi comunicativi, e più in generale ai sistemi di comunicazione visuali. Ha vinto il premio Compasso d'Oro (1995) come co-autore del catalogo multimediale per il Museo Poldi Pezzoli.

È autrice, fra l'altro, dei volumi La messa in scena del prodotto. Packaging: identità e consumo (2002) e Packaging design. Storia, linguaggi, progetto (2005). È curatrice di Altre figure. Intorno alle figure di argomentazione (2011); Anticorpi comunicativi. Progettare per la comunicazione di genere (2012); Design e dimensione di genere. Un campo di ricerca e riflessione tra culture del progetto e culture di genere (2015); Un'interfaccia per il welfare. Le funzioni sociali del design della comunicazione (2017); Progetto e culture visive. Elementi per il design della comunicazione (2018). 